\title{
The distinct vibrational signature of grain-boundary water in nano-crystalline ice films
}

\author{
*Takahiro Kondo ${ }^{1,2}$, Hiroyuki S. Kato ${ }^{2}, * *$ Maki Kawai $^{2,3}$ and Mischa Bonn ${ }^{4}$ \\ ${ }^{1}$ Institute of material science, University of Tsukuba, 1-1-1 Tennodai, Tsukuba, Ibaraki, 305-8573 Japan \\ ${ }^{2}$ Surface Chemistry Laboratory, RIKEN (The Institute of Physical and Chemical Research), 2-1-1, Wako, Hirosawa, \\ Saitama, 351-0198 Japan \\ ${ }^{3}$ Department of Advanced Materials Science, University of Tokyo, Kashiwa, Chiba 277-8561, Japan \\ ${ }^{4}$ FOM Institute for Atomic and Molecular Physics, Kruislaan 407, 1098 SJ Amsterdam, The Netherlands \\ *takahiro@riken.jp , **maki@riken.jp
}

\begin{abstract}
We have characterized the vibrational response of grain-boundary water in crystalline ice films consisting of nanometer-sized crystalline domains. The crystalline ice is grown isothermally on $\mathrm{Ru}(0001)$, and the crystalline domain size is controlled by varying the crystallization temperature. A distinct vibrational feature in the OD stretching region is observed, which we attribute to grain-boundary water. The relative contribution of the grain boundary vibrational response to the overall spectrum increases with decreasing crystal domain size. Its central frequency is located around $2500 \mathrm{~cm}^{-1}$, between that of amorphous solid water and crystalline ice.
\end{abstract}


The characterization of the grain boundaries of ice crystals is important for understanding a wide variety of environmental subjects such as electrification of clouds, ${ }^{1,2}$ phenomena such as soil freezing, frost heave and perma-frost ${ }^{3,4}$ as well as fundamental subjects such as the sintering, coarsening, transport behavior and many other properties of bulk ice. ${ }^{5-9}$ The understanding of crystalline grain boundaries is also of importance for chemical reactions on ice, such as the formation of prebiotic organic molecules in the interstellar medium ${ }^{10}$ and reactions that lead to ozone depletion in the stratosphere. ${ }^{11}$ Ice grains grown on a solid surface always have a finite grain size, which depends on the condition of the preparation., 6, 12 Thus, in addition to the surface of ice exposed to the vacuum, there exist many grain-grain interfaces. Molecules and/or ions that can react on ice may diffuse over the surfaces and interfaces of ice grains; bulk diffusion is much less likely from the viewpoint of thermodynamics. The interaction between such molecular moieties and the ice surface/interface (i.e. water molecules at the grain-boundary) therefore determine the fate of these molecules. As a result, the behavior of the molecular monolayer of water at the surface of ice grains and clusters has received much attention. Particularly noteworthy are infrared (IR) spectroscopic studies as sensitive and non-destructive methods to characterize the vibrational properties of aqueous solids. Indeed, Devlin, Buch, and co-workers have extensively investigated size-selected water clusters in the gas-phase by IR spectroscopy and have found specific vibrational response of water at the surface and right below the surface (sub-surface) in small water clusters (Ref.13, 14 and references therein). Such information has not yet been obtained for water at the interfaces between crystalline grains in solid films.

Here, we report the vibrational response of grain-boundary water molecules between crystalline grains at temperatures around $150 \mathrm{~K}$. We have prepared well-defined nanometer-sized crystalline ice (CI) grains by the isothermal crystallization of amorphous solid 
water (ASW) films grown on a Ru(0001) surface. The number and size of the CI grain can be controlled in our isothermal crystallization experiments through the different temperature dependences of the nucleation rate and growth rate of the CI in the bulk of the ASW films. ${ }^{5,12 \text {, }}$ ${ }^{15-18}$ Our results reveal a distinct vibrational response of grain boundary water molecules localized around the nanometer-sized CI grains.

The experimental apparatus used in this work has already been described elsewhere. ${ }^{17-19}$ Briefly, the infrared light from the Fourier transform IR spectrometer (JASCO FT/IR-550) was $p$ polarized through a ZnSe polarizer. It was then focused on the sample surface in the ultra-high vacuum (UHV) chamber by a concave mirror through a BaF viewport at an $85^{\circ}$ grazing angle of incidence. The IR light reflected from the sample was detected by a mercury-cadmium-telluride (MCT) detector. The light paths outside the UHV chamber were purged by pure nitrogen gas to avoid absorption by ambient air, which contains $\mathrm{CO}_{2}$ and $\mathrm{H}_{2} \mathrm{O}$. IRAS spectra were recorded at 4 $\mathrm{cm}^{-1}$ resolution with 20 scan (40 s) averages. The IR absorbance $A$ is defined as $A=-\ln \left(R / R_{0}\right)$, where $R$ and $R_{0}$ are the reflected intensities with and without the water layers on the substrate, respectively. We investigated fully deuterated water $\left(\mathrm{D}_{2} \mathrm{O}\right)$ because the IR-spectrometer has a higher sensitivity in the O-D stretch frequency range than in the O-H stretch range of $\mathrm{H}_{2} \mathrm{O}$.

The $\mathrm{Ru}(0001)$ substrate was cleaned using standard sputtering, annealing, oxidation and flashing cycles in the UHV chamber with a base pressure of $\sim 1 \times 10^{-10}$ Torr. The temperature of the sample surface is measured by C-type (W5\%Re-W26\%Re) and K-type (Alumel- Chromel) thermocouples spot-welded to the edge of crystal. Each of the output Seebeck voltages is measured by a digital multimeter, referenced to liquid nitrogen temperature. The substrate temperature was then carefully calibrated by temperature-programmed desorption (TPD) measurements of $\mathrm{D}_{2} \mathrm{O}$ from $\mathrm{Ru}(0001)$ prior to the experiment. ${ }^{20}$ 
The degree of thickness of the water layer is expressed here in monolayers (ML). 1 ML is determined by the desorption amount of the first water layer strongly interacting with the $\mathrm{Ru}(0001)$ surface that gives rise to one desorption feature near $180 \mathrm{~K}$ in the thermal desorption experiments; ${ }^{20}$ this feature saturates with exposure, after which multilayer desorption occurs near $160 \mathrm{~K}$. Thin films of ASW of $\sim 50$ monolayers (ML) were deposited on the surface at $\sim 90$ K. After the deposition of ASW, the layers were heated to the designated isothermal temperature, $T$, where IRAS spectra were recorded continuously at $40 \mathrm{~s}$ intervals. The IRAS spectra measured during the ASW crystallization can be reproduced very well by a linear combination of the spectrum at $t=0$ (amorphous solid water) and the spectrum after staying at temperature $T$ sufficiently long that the spectral response does not change any longer (crystalline ice with possible contributions form grain boundary water, see below). The "converted fraction” is defined here as the long-time contribution divided by the weighted sum of the two. ${ }^{17,18,21}$ The average CI grain size in the converted CI films was controlled by varying $T$ owing to the different activation energies for nucleation and growth; At high $T$, nucleation is dominant and many small crystalline grains appear, whereas at low $T$ the nucleation probability is low and relatively large (tens of $\mathrm{nm}$ ) crystalline grains appear in the films. ${ }^{5,12,15-18}$

Fig. 1 shows typical IRAS spectra of ASW and CI films on Ru(0001), where each spectrum is normalized by the spectrally integrated intensity in the O-D stretch region. An absorption band with a large width at $2400-2600 \mathrm{~cm}^{-1}$ in Fig. 1 is typical of the OD stretching vibrational mode $\left(v_{\mathrm{OD}}\right)$ of $\mathrm{D}_{2} \mathrm{O}$ forming a hydrogen bond network, where intramolecular and intermolecular coupling among $\mathrm{D}_{2} \mathrm{O}$ molecules cause a large peak-width of $\mathrm{v}_{\mathrm{OD}}{ }^{14,22}$ The first moment of the absorbance peak is located at higher frequency for ASW: after crystallization the peak shifts to lower frequency due to the well-ordered and more strongly hydrogen-bonded 
network of CI, as shown in Fig. 1.

As shown in Fig. 1(b) in detail, the spectrum of CI consists of three major noticeable absorption peaks at $\sim 2340, \sim 2440$ and $\sim 2500 \mathrm{~cm}^{-1}$. With increasing crystallization temperature $T$, the high-frequency peak shoulder increases in amplitude relative to the low-frequency shoulder, as indicated by the arrows in Fig. 1(b). We attribute this effect to the variations in average crystal size with varying temperature. This observation is reminiscent of previous TPD studies, ${ }^{15}$, ${ }^{16}$ where differently shaped TPD spectra of probe molecules desorbing from CI surfaces were observed, depending on the crystallization conditions. The difference of the TPD shape was attributed to the residual non-crystallized component surrounding the annealed CI grains, which resists conversion to $\mathrm{CI}^{23,24}$ As will be described below, our results provide new insight into the nature of this residual component: it is shown to be distinctly different from the amorphous phase, and we identify it as grain boundary water.

We note two possible effects ${ }^{25,26}$ that may complicate a straightforward interpretation of the IRAS spectra: Fresnel (local field) effects and Mie scattering. Fresnel effects may be different for the different films due to morphological effects caused by the non-uniformity of water in the film. Mie scattering effects may vary as the crystal size varies with temperature, changing the scattering properties of the film. We can, however, rule out significant contributions of these two effects by close inspection of the experimental data recorded at 156.5 and $166.5 \mathrm{~K}$, respectively.

At $T=156.5 \mathrm{~K}$, we have shown previously, using He atom scattering, that the film morphology changes occur already during crystallization. ${ }^{17,18}$ The changes in the IRAS spectra with time are therefore expected to be predominantly due to a change in the average crystal size with time. Following crystallization, the CI grains are expected to decrease in size by factor of $\sim 2.5$ for the spectra shown in Fig. 2(a). This factor is determined from the decrease of the total 
amount of water. The resulting changes in the IRAS spectrum are small as evident from the normalized spectra shown in Fig. 2c: no significant spectral changes are observed, as shown in the inset in Fig. 2c, implying that Mie scattering effects [25,26] are not significant. Clearly, the spectral changes are very small compared to the temperature-dependent differences shown in Fig. 1. In the latter, moreover, for the smaller crystal grains produced at higher temperature [5, 12, 15-18], the shoulder at $\sim 2500 \mathrm{~cm}-1$ is increased contrary to the trend of Mie-scattering. It therefore seems evident that the temperature-dependent changes in the IRAS spectrum (Fig. 1) cannot be explained simply by changes in Mie scattering due to variations in the crystal size.

The effect of morphological changes can be ruled out by inspecting the time-dependent spectra at $166.5 \mathrm{~K}$ shown in Fig. 2(b). At this temperature, morphological changes and exposure of the first water layer to the vacuum occur while substantial CI is already present on the surface, as we have shown previously using He atom scattering. ${ }^{17,}{ }^{18}$ The inset in Fig. 2(b) is the corresponding result of $\mathrm{He}$ atom scattering, where the increases in the He signal at $~ 300 \mathrm{~s}$ signifying morphological changes occurs long after crystallization at $20 \mathrm{~s}$. A close inspection of the spectra as a function of time again reveals very small changes in the IR response due to morphological changes as shown by corresponding blue and red IR spectrum in Fig. 2(d). Hence we conclude that changes in the film morphology also do not significantly affect our conclusions. We note that the separation between spectral effects due to film morphology and crystallite size made in the above argumentation is somewhat artificial; we can therefore not conclusively exclude any possible effects due to local field and/or Mie scattering effects, but the results of Fig. 2 indicate that these effects are relatively small.

The crystallization of ASW layers proceeds by random nucleation in the bulk of the material followed by an effectively homogeneous growth, with simultaneous sublimation of the water occurring. ${ }^{12,17,18}$ As shown in Fig. 3(a), the rate of crystallization depends critically on 
temperature $T$. The time traces can be reproduced very well by a calculation based on the advanced Avrami theory. ${ }^{27}$ This analysis, shown as lines in Fig. 3(a), provides us with both the growth rate $G(T)$ and the nucleation probability $J_{N}(T)$ of crystalline nuclei at each isothermal temperature $T .^{17,18}$ The derived nucleation probability, $J_{N}(T)\left[\mathrm{ML}^{-3} \mathrm{~s}^{-1}\right]$ is in good agreement with previous work. ${ }^{15,16}$ Its value allows us to estimate the average grain radius of the transformed CI, $R_{C I}[\mathrm{ML}]$, shown in Fig. 3(b) at each temperature, using the simple equation:

$$
R_{C I} \approx 0.5\left[J_{N}(T) \int_{0}^{\infty}(1-\chi(t)) d t\right]^{-1 / 3}
$$

where $\chi(t)$ is the time-dependent fraction of the conversion from ASW to CI at the time $t$, as shown in Fig 3a. This formula can be understood as follows: the integral provides the average volume of the amorphous water during the period. The average unit grain volume can be obtained by multiplying the integral with $J_{N}$, in essence dividing the total volume by the number of nucleation cores. Another way of understanding the formula is that the integral provides the average time that a water molecule remains in the amorphous phase, so that the formula provides the average distance between nucleation events. The factor of 0.5 is to convert diameter to radius. The numerical integration of the traces in Fig. 3(a) reveal that the estimated grain radius decreases from $\sim 18 \mathrm{ML}$ to $\sim 1.9 \mathrm{ML}$ with increasing isothermal temperature from 152.5 K to $168 \mathrm{~K}$, as shown in Fig. 3(b).

The average calculated grain diameter at low temperatures ( $\sim 35 \mathrm{ML})$ is comparable to the initial film thickness (50 ML). Hence the contribution to the spectrum from the residual water at the grain boundary in these CI films will be very small. Thus we assign the spectrum at $T=152.5 \mathrm{~K}$ ( $\sim 35 \mathrm{ML}$ grain size) to that of ‘pure’ CI, i.e., CI grains with only negligible residual water at the grain-grain boundary. By subtracting this 'pure' CI spectrum component from measured IR spectrum in Fig. 1, the spectrum of the residual water at the grain boundary can be obtained. The amount of 'pure' CI spectrum that has to be subtracted was determined by 
calculating the surface boundary area using the experimentally derived average grain size, $R_{C I}$, in Fig. 3(b). The homogeneous growth of crystalline ice nuclei results in quasi-sphere ice grains. Assuming complete contact among the ice grain boundaries, the ice grain boundary can be considered as the Voronoi cell of the ice spheres. In the case of closed pack sphere, the Voronoi cell is a rhombic dodecahedron as the Wigner-Seitz unit cell of a face centered cubic lattice. Then, we can easily estimate the fraction of water molecules $S$ contained in the outer monolayer at the surface of a rhombic dodecahedron (Voronoi cell of the sphere with radius of $R_{C I}$ ), i.e. the water in the shell between radius $R_{C I}-0.5$ and $R_{C I}$ (note that $R_{C I}$ is expressed in water monolayers $\left.^{21}\right)$ :

$$
S=\frac{4 \sqrt{2} R_{C I}{ }^{3}-4 \sqrt{2}\left(R_{C I}-0.5\right)^{3}}{4 \sqrt{2} R_{C I}{ }^{3}}=\frac{R_{C I}{ }^{3}-\left(R_{C I}-0.5\right)^{3}}{R_{C I}{ }^{3}}
$$

The derived series of the IRAS difference spectra of the residual water molecules surrounding the CI grain (referred to as 'grain boundary water') is shown in Fig. 4(a) with calculated value of $S$ as a function of $T$. Here, the deconvolution is conducted without inclusion of the spectrum components of the interface between ice and substrate (and thus the component of the first water layer) as well as ice-vacuum interface since these spectrum components are expected to be much smaller than the other components of CI, ASW and grain boundary. The relative contribution of grain boundary water increases as the average grain size decreases. For the smallest crystalline grains at the highest temperatures, it amounts to as much as $60 \%$ of the overall spectrum. The application of equation (2) is justified by the observation that shapes of the thus derived grain boundary spectra at different $T$ (Fig. 4(a)) are indistinguishable (i.e. independent of the isothermal temperature) as shown by the normalized spectrum in Fig. 4(b) and also markedly different from the shape of ASW and CI spectra. Different procedures to extract this 'grain boundary spectrum' from the raw data result in very similar spectra. 
The spectral shape of the grain boundary water differs from that of CI and ASW in Fig. 4, which indicates the existence of the water molecules having a different vibrational property from that of ASW and CI: the residual water molecules surrounding CI are not just unconverted ASW but exhibit distinct vibrational properties. Note that the vibrational response is markedly different not only from ASW or CI but also from that of water at the ice-air interface, which is characterized by a dangling O-D bond with a mode frequency around $\sim 2700 \mathrm{~cm}^{-1} .{ }^{14,28}$ The spectral shape of the grain boundary water is relatively narrower than that of ASW and CI and the central frequency is located around $2500 \mathrm{~cm}^{-1}$, between that of ASW and CI as shown in Fig. 4, indicating a well-ordered H-bonded network with intermediate strength of two phases of ASW and CI. This is also different from previously reported spectrum components of disordered subsurface ice. ${ }^{13,14}$ The observation of a well-defined, relatively sharp vibrational spectrum is surprising considering the heterogeneity one might expect for this interfacial system. As the future work, the diffusivity of foreign molecule/atom along the ice grain surfaces may be derived and discussed in detail by tracing the spectrum component of the grain boundary water.

In summary, we have experimentally derived the vibrational spectrum of localized grain-boundary water molecules surrounding nanometer-sized ice crystallites isothermally grown on $\mathrm{Ru}(0001)$. A distinct vibrational response of the OD stretching vibrational mode of grain-boundary water is reported, located in the frequency region around $2500 \mathrm{~cm}^{-1}$, between the amorphous solid water and CI, with a relatively sharp width. This is different from known absorption frequencies of $v_{\mathrm{OD}}$ (dangling-OD) at 2670,2730 and $2750 \mathrm{~cm}^{-1}$, possibly due to the interaction among the grain boundary water molecules.

\section{Acknowledgement}

T. K. appreciates the financial support from the SPR System in RIKEN (2003-2005). M.B. 
is grateful for support from the Young Academy of the Royal Dutch Academy of Sciences. This research was also partially supported by RIKEN Research Program "Nanoscale Science and Technology Research” and by the Ministry of Education, Culture, Sports, Science and Technology (MEXT), through “Grant-in-Aid for Young Scientists (B) 17760034, 2005-2007”.

\section{References}

1. G. J. Turner and C. Stow, Philos. Mag. A 49, L25 (1984).

2. J. G. Dash, B. L. Mason and J. S. Wettlaufer, J. Geophys. Res. 106, 20395 (2001).

3. J. G. Dash, H.-Y. Fu, and J. S. Wettlaufer, Rep. Prog. Phys. 58, 115 (1995).

4. J. S. Wettlaufer, Philos. Trans. R. Soc. London, Ser. A 357, 3403 (1999).

5. "Physics of ice” Edited by V. F. Petrorenko et al. (Oxford University press, 1999).

6. M. Chaplin, “Water structure and science” http://www.lsbu.ac.uk/water/

7. J. G. Dash, Rev. Mod. Phys. 71, 1737 (1999).

8. L. Benatov and J. S. Wettlaufer, Phys. Rev. E. 70, 061606 (2004).

9. J. G. Dash, A. W. Rempel and J. S. Wettlaufer, Rev. Mod. Phys. 78, 695 (2006).

10. P. Ehrenfreund, W. Irvine, L. Becker, J. Blank, J. R. Brucato, L. Colangeli, S. Derenne, D. Despois, A. Dutrey, H. Fraaije, A. Lazcano, T. Owen and F. Robert, Rep. Prog. Phys. 65, 1427 (2002)

11. J. P. D. Abbatt, Chem. Rev. 103, 4783 (2003).

12. "Water in Confining Geometrie”, edited by V. Buch and J. P. Devlin (Springer-Verlag, Berlin, 2003).

13. V. Buch, S. Bauerecker, J. P. Devlin, U. Buck, and J. K. Kazimirski, Int. Rev. Phys. Chem. 23, 375 (2004).

14. J. P. Devlin, J. Geo. Res. 106, 33333 (2001). 
15. Z. Dohnálek, G. A. Kimmel, R. L. Ciolli, K. P. Stevenson, R. S. Smith, and B. D. Kay, J. Chem. Phys. 112, 5932 (2000).

16. D. J. Safarik, R. J. Meyer, and C. B. Mullins, J. Chem. Phys. 118, 4660 (2003).

17. T. Kondo, H. S. Kato, M. Bonn and M. Kawai, J. Chem. Phys. 126, 181103 (2007).

18. T. Kondo, H. S. Kato, M. Bonn and M. Kawai, J. Chem. Phys. 127, 094703 (2007).

19. T. Kondo, H. S. Kato, T. Yamada, S. Yamamoto and M. Kawai, Eur. Phys. J. D 38, 129 (2006).

20. T. Kondo, S. Mae, H. S. Kato and M. Kawai, Surf. Sci. 600, 3570 (2006).

21. E.H.G. Backus, M. Grecea, A.W. Kleyn, and M. Bonn, Phys. Rev. Lett. 92, 236101 (2004).

22. W. Hagen, A. G. G. M. Tielens and J. M. Greenberg, Chem. Phys. 56, 367 (1981).

23. P. Jenniskens and D. F. Blake, Science 265, 753 (1994).

24. P. Jenniskens and D. F. Blake, Astrophys. J. 473, 1104 (1996).

25. S. Mitlin and K. T. Leung, J. Phys. Chem. B 106, 6234 (2002).

26. S. Haq and A. Hodgson, J. Phys. Chem. C 111, 5946 (2007).

27. E. H. G. Backus and M. Bonn, J. Chem. Phys. 121, 1038 (2004).

28. J. P. Devlin and V. Buch, J. Phys. Chem. B 101, 6095 (1997)

Figure caption

Fig. 1: Typical IRAS spectra of ASW ( 25 ML), (a), crystalline ice, (b). The spectra were normalized by the integral intensity of the absorbance.

Fig. 2: (a) and (b) : Evolution of IRAS spectra upon annealing at (a) $156.5 \mathrm{~K}$ and (b) $166.5 \mathrm{~K}$ for initially 48 and 61.5 ML ASW on Ru(0001), respectively. During the period up to $t=0 \mathrm{~s}$, the temperature increases from $\sim 90 \mathrm{~K}$ to the designed temperature with a heating rate of $\sim 0.2 \mathrm{~K} / \mathrm{s}$. The temperature is then held at the designated temperature. The absorbance intensity decreases 
with time due to the sublimation of water. ${ }^{17,} 18$ The inset in (b) shows the simultaneously measured scattered He intensity (specularly reflected from the surface) as a function of time at $T$ $=166.5 \mathrm{~K}$. The initial rapid increase in the HAS intensity indicates the crystallization of ASW while second increase at $\sim 300 \mathrm{~s}$ indicates the exposure of the first water layer to the vacuum. ${ }^{17}$, ${ }^{18}$ (c) and (d) show the normalized IRAS spectra shown in (a) and (b), respectively, at the points designated by the arrows. The spectra are normalized based on the absorption peak intensity at $\sim 2450 \mathrm{~cm}^{-1}$ with scaling factors indicated in the figure. The blue and red IR spectra in (d) correspond to spectra before and after the morphological changes at $\mathrm{t}=300 \mathrm{~s}$ apparent from the He scattering (b).

Fig, 3: (a) Converted fraction from ASW to CI estimated from IRAS fitting analysis shown in Fig. 1(b). ${ }^{17,18}$ Results at various temperatures are shown as a function of isothermal annealing time. Solid curves are indicating the calculated results based on the model presented in Ref. 27. (b) Estimated average CI grain size as a function of the isothermal annealing temperature

Fig. 4: (a) Derived IRAS spectra of water molecules at grain-boundary of CI (see text). (b) Normalized spectrum of grain boundary water shown in (a) and average spectrum of normalized spectra. The spectrum of ASW and CI (spectrum at $T=152.5 \mathrm{~K}$ ) are also shown for the comparison. 
Chemical Physics Letters

Fig. 1 Takahiro Kondo, Hiroyuki S. Kato, Maki Kawai, and Mischa Bonn

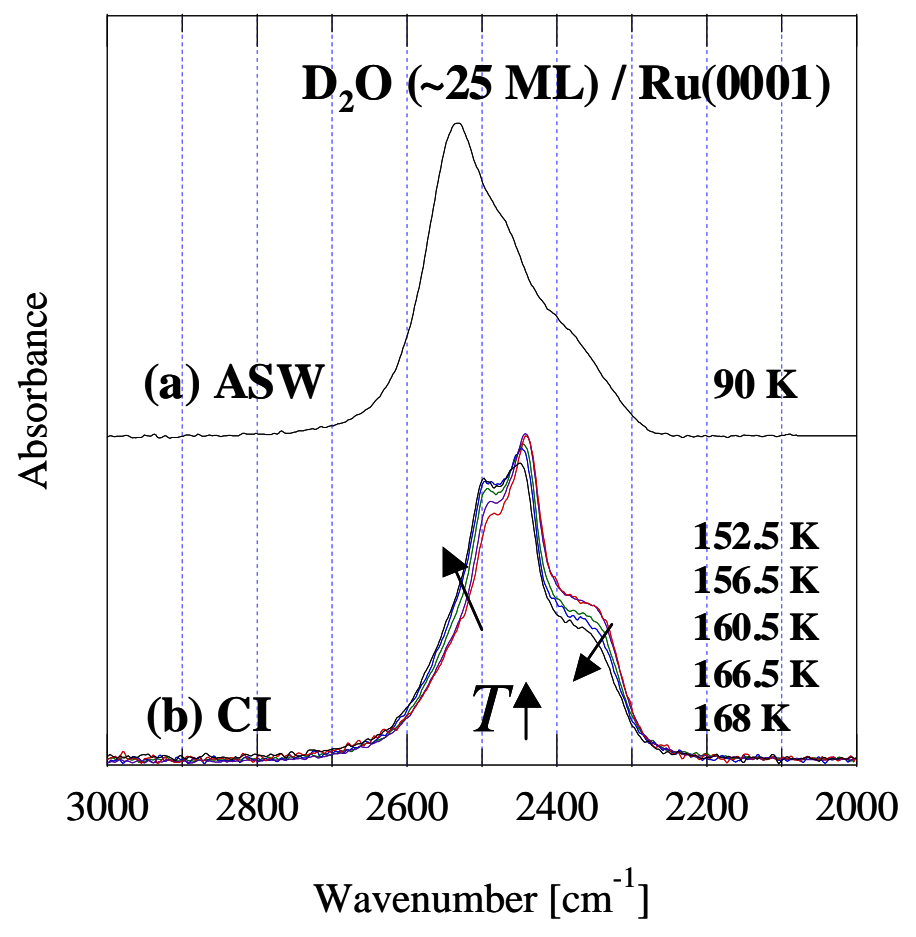


Chemical Physics Letters

Fig. 2 Takahiro Kondo, Hiroyuki S. Kato, Maki Kawai, and Mischa Bonn

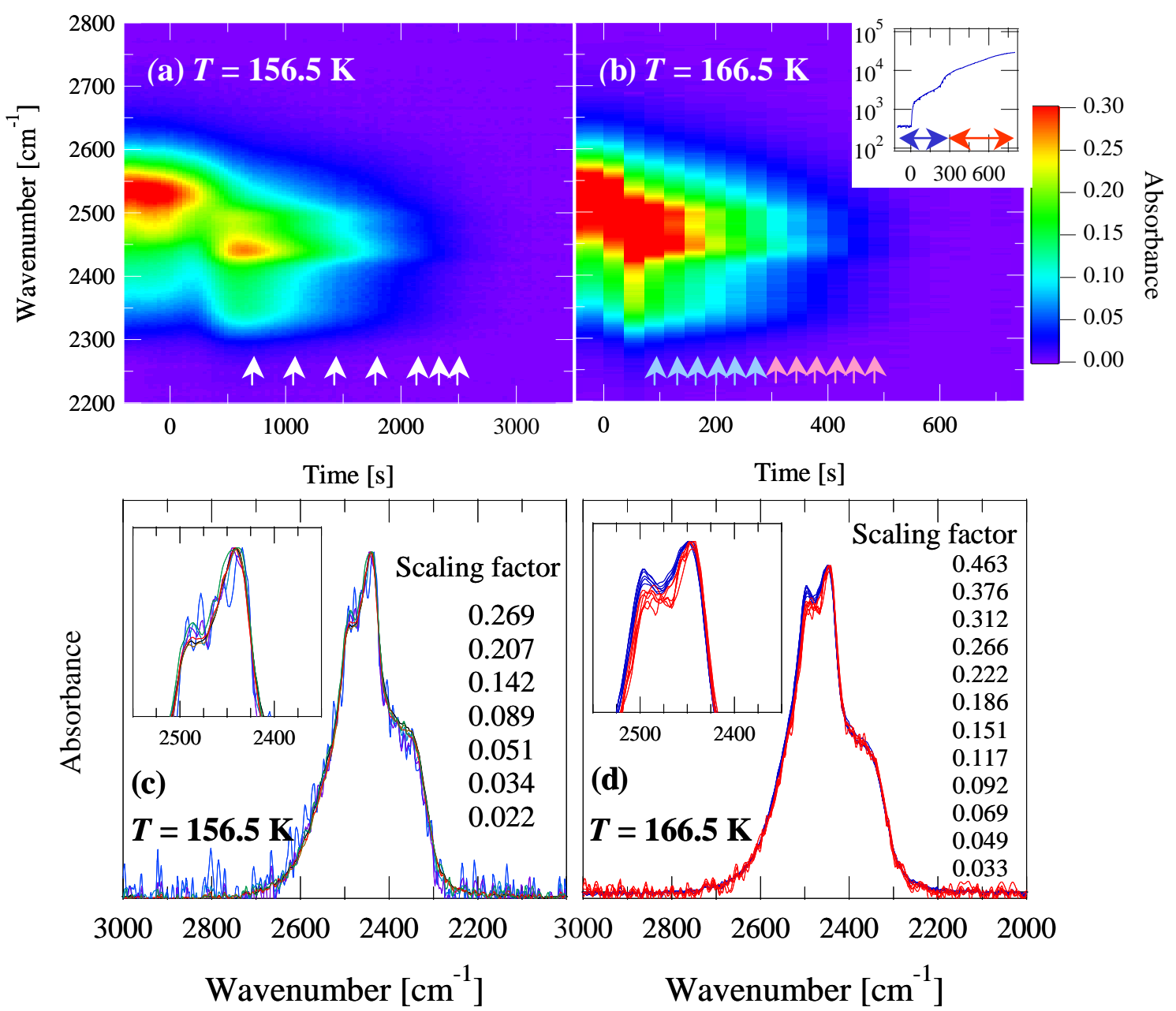


Chemical Physics Letters

Fig. 3 Takahiro Kondo, Hiroyuki S. Kato, Maki Kawai, and Mischa Bonn
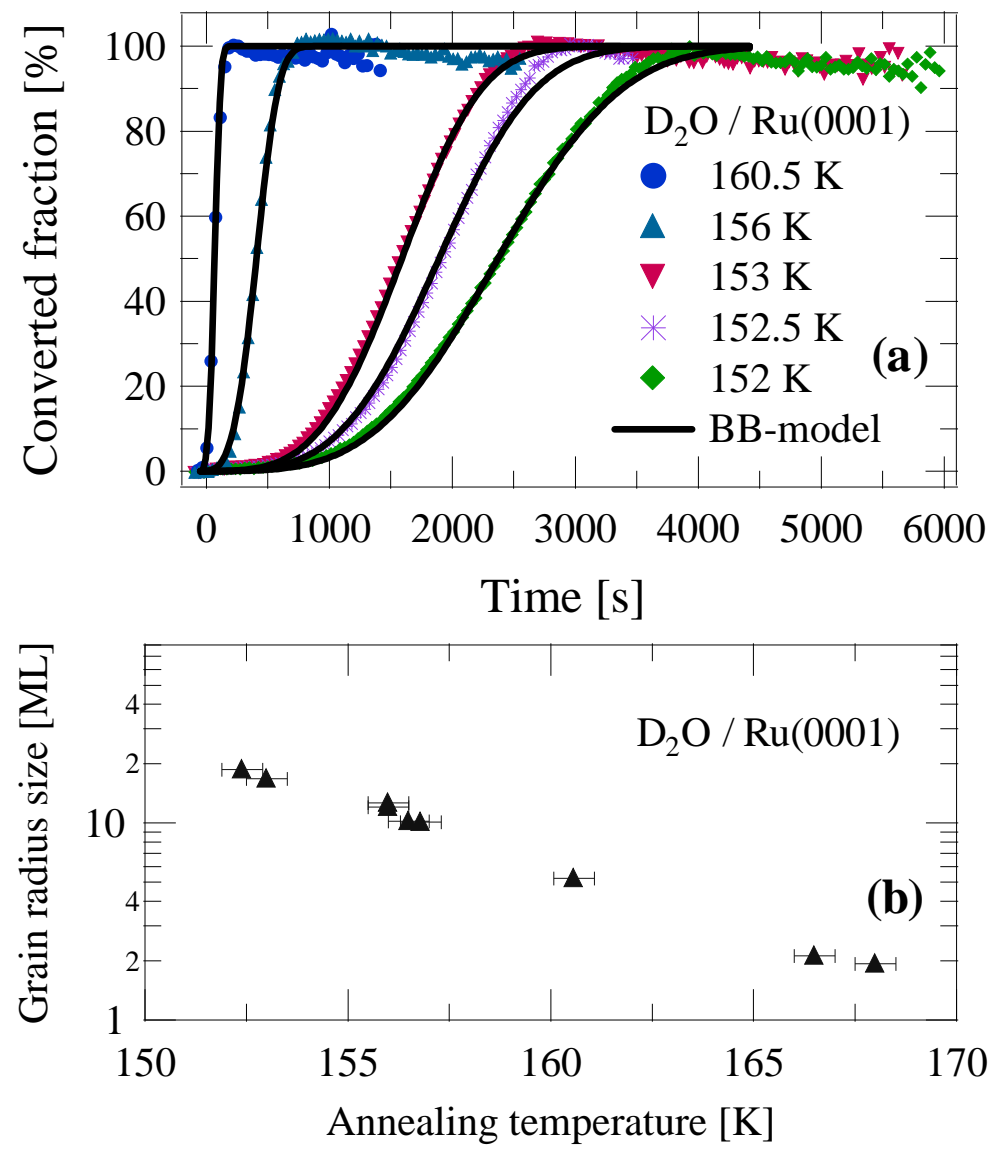
Chemical Physics Letters

Fig. 4 Takahiro Kondo, Hiroyuki S. Kato, Maki Kawai, and Mischa Bonn

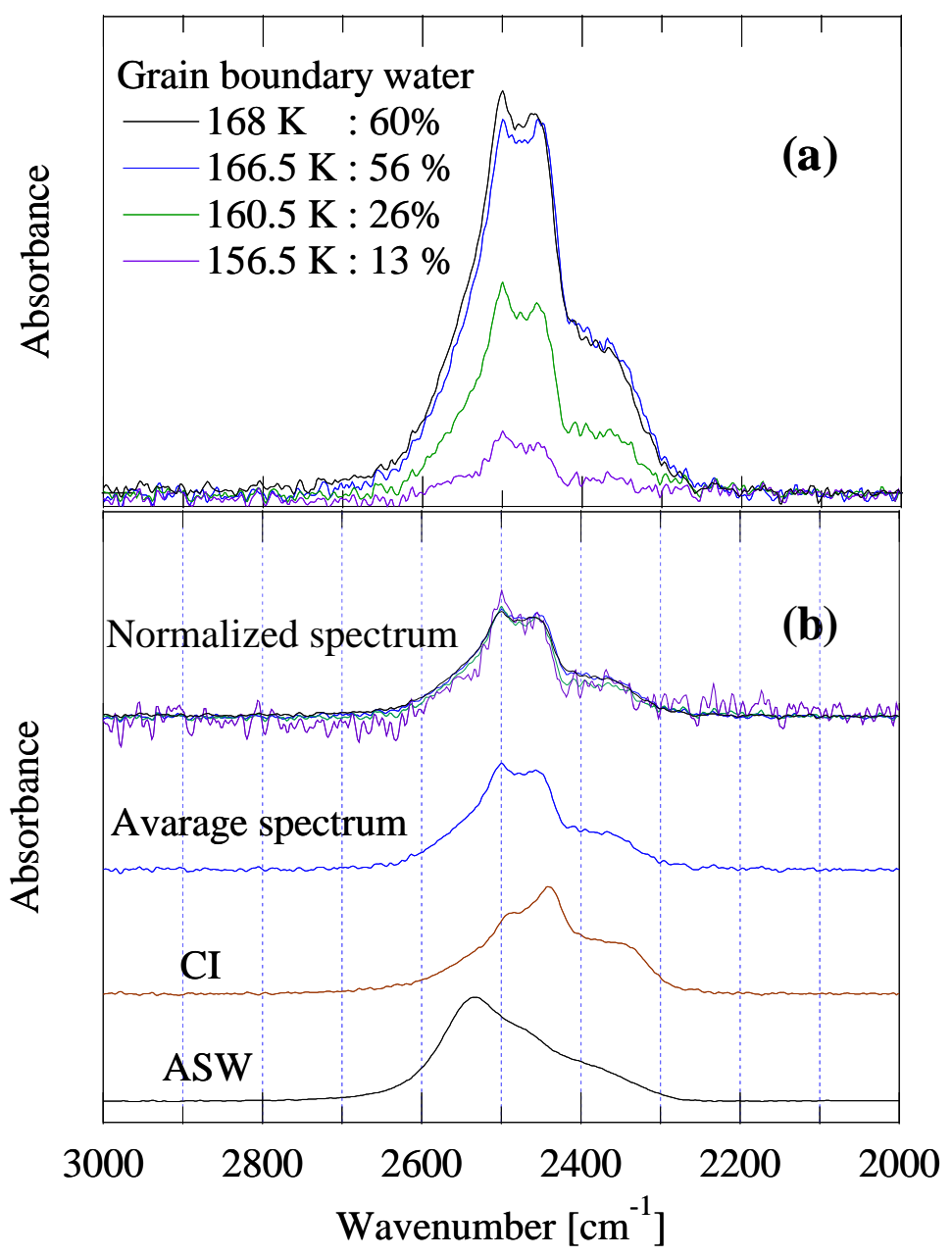

